\title{
Sistemas integrados de produção agropecuária e os efeitos do componente animal
}

\author{
Integrated agricultural production systems and the effects of the animal component \\ Sistemas integrados de producción agrícola y efectos del componente animal
}

\section{Resumo}

Grande parte do rebanho de bovinos do Brasil se encontra em sistemas a pasto em áreas intertropicais, como a Amazônia. Visto a necessidade de buscar maior sustentabilidade ambiental e econômica da pecuária, este trabalho teve como objetivo discutir a introdução e os benefícios do componente animal em sistemas integrados de produção agropecuária. A revisão baseou-se no referencial da pesquisa bibliográfica coletados através do levantamento das produções científicas sobre sistemas integrados de produção agropecuária (SIPA), produzidas entre os anos de 2000 a 2020. Os SIPAs consistem na implantação de diferentes atividades (pecuária, agricultura e silvicultura) na mesma área, em consórcio, sequencial ou rotacionado, manejados com ênfase no contexto de produção e sustentabilidade. A inclusão do componente animal traz diversos benefícios para o sistema, seja pelo estímulo à produção de biomassa, aumento da ciclagem de nutrientes, aumento da massa e diversidade microbiana, melhoria nas condições físicas, químicas e da fertilidade do solo. Além de beneficiar o sistema, o componente animal se beneficia com a inclusão, especialmente pelos melhores valores nutricionais das forrageiras e conforto térmico oferecido por alguns arranjos. Outro fator benéfico dos sistemas integrados para a pecuária, seria a sua utilização em métodos alternativos na recuperação e renovação de pastagens em áreas degradadas. Diversas são as modalidades/arranjos que podem ser empregados na integração, em que são montados visando aproveitar as sinergias existentes entre as atividades, as quais, possuem seus benefícios particulares ou mútuos quando bem conduzidos.

Palavras-chave: Bem estar animal; Bovinocultura; Estresse térmico; Sustentabilidade.

\section{Abstract}

A large part of the cattle herd in Brazil is found in pasture systems in intertropical areas, such as the Amazon. Given the need to seek greater environmental and economic sustainability of livestock, this work aimed to discuss the introduction and benefits of the animal component in integrated systems of agricultural production. The review was based on the references of bibliographic research collected through the survey of scientific production on integrated 
systems of agricultural production (SIPA), produced between the years 2000 to 2020. SIPAs consist of the implementation of different activities (livestock, agriculture and forestry) in the same area, in consortium, sequential or rotated, managed with an emphasis on the context of production and sustainability. The inclusion of the animal component brings several benefits to the system, whether by stimulating biomass production, increasing nutrient cycling, increasing microbial mass and diversity, improving physical, active and soil fertility conditions. In addition to being a beneficiary of the system, the animal component benefits from inclusion, especially due to the better nutritional values of forages and the comfort offered by some arrangements. Another beneficial factor of integrated systems for livestock would be their use in alternative methods in the recovery and renewal of pastures in degraded areas. There are several modalities/arrangements that can be used in the integration, in which basics are set up as existing synergies between activities, which have their particular or mutual benefits when properly conducted.

Keywords: Animal welfare; Cattle farming; Thermal stress; Sustainability.

\section{Resumen}

Una gran parte del rebaño de ganado en Brasil se encuentra en sistemas de pastos en áreas intertropicales, como el Amazonas. Dada la necesidad de buscar una mayor sostenibilidad ambiental y económica de la ganadería, este trabajo tuvo como objetivo discutir la introducción y beneficios del componente animal en los sistemas integrados de producción agrícola. La revisión se basó en las referencias de la investigación bibliográfica recopilada a través de la encuesta de producción científica sobre sistemas integrados de producción agrícola (SIPA), realizada entre los años 2000 a 2020. Los SIPA consisten en la implementación de diferentes actividades (ganadería, agricultura y silvicultura) en la misma área, en consorcio, secuencial o rotado, manejado con énfasis en el contexto de producción y sustentabilidad. La inclusión del componente animal trae varios beneficios al sistema, ya sea estimulando la producción de biomasa, aumentando el ciclo de nutrientes, aumentando la masa y diversidad microbiana, mejorando las condiciones físicas, activas y de fertilidad del suelo. Además de ser beneficiario del sistema, el componente animal se beneficia de la inclusión, especialmente por los mejores valores nutricionales de los forrajes y la comodidad que ofrecen algunos arreglos. Otro factor beneficioso de los sistemas integrados para la ganadería sería su uso en métodos alternativos en la recuperación y renovación de pastos en áreas degradadas. Existen varias modalidades / arreglos que se pueden utilizar en la integración, en los cuales los conceptos básicos se configuran como sinergias existentes entre actividades, que tienen sus beneficios particulares o mutuos cuando se llevan a cabo correctamente.

Palabras clave: Bienestar animal; Criar ganado; Estrés termal; Sustentabilidad.

\section{Introdução}

A necessidade de sistemas que garantam a produção de alimentos por vias sustentáveis, fez retornar a prática de sistemas integrados de produção agropecuária (SIPA). Atualmente, os modelos possuem o foco na integração e manejo de componentes produtivos, gerando e aumentando a sinergia entre as áreas integradas. Apesar dos diferentes arranjos de sistemas o foco principal é a intensificação sustentável do processo (Moraes et al., 2018; Carvalho et al., 2018b).

Os sistemas integrados de produção agropecuária consistem na implantação de diferentes atividades (pecuária, agricultura e silvicultura) na mesma área, em consórcio, sequencial ou rotacionado, aproveitando as sinergias existentes entre elas. A interação dos componentes destas atividades requer um manejo desafiador, devido à dinâmica e a complexidade dos sistemas. Portanto, a integração favorece a utilização mais efetiva de todos os fatores bióticos e abióticos de produção, aumentando assim a produtividade e a rentabilidade (Lima \& Gama, 2018; Soares et al., 2018).

A combinação do componente animal sobre pastagens em áreas de produção agrícola e silvicultura, representa uma oportunidade de gerar renda e trazer benefícios em relação aos sistemas tradicionais. Aproximadamente dois terços do rebanho da pecuária brasileira ficam localizados em zona intertropical e com sistemas de produção quase que exclusivamente dependentes das pastagens, o que incita alguns dos principais fatores que afetam o desempenho dos animais, de forma geral, a deficiência nutricional e estresse térmico calórico. Devido a esses fatores, a inserção do componente animal em áreas de integração, pode ser viabilizada, especialmente, devido os melhores valores nutricionais e de conforto térmico oferecido por alguns arranjos de sistemas de integração (Soares et al., 2018; Alves \& Karvatte, 2019).

No período compreendido entre 1975 a 2017, o rebanho nacional passou de 101.673.753 para 171.858.168 animais, aumentando significativamente a necessidade de áreas de pastagens (Instituto Brasileiro de Geografia e Estatística [IBGE], 2018). Cerca de $77 \%$ deste crescimento ocorreu na região Amazônica, e está associado ao desmatamento da mesma, pelas 
antigas práticas de manejo, que atualmente não são mais apropriadas, visto que novas práticas agropecuárias vêm sendo implantadas, possibilitando obter maior produtividade sem a necessidade de extensão de novas áreas (IBGE, 2018; Ohashi et al., 2018; Dias-Filho \& Lopes, 2020). Considerando os benefícios potenciais do sistema de integração para o componente animal, este trabalho teve por objetivo discutir a inclusão do componente animal em sistemas integrados, bem como os benefícios deste sistema para os animais.

\section{Metodologia}

O presente trabalho baseou-se no referencial da pesquisa bibliográfica, cujos dados foram coletados através do levantamento das produções científicas sobre sistemas integrados de produção agropecuária (SIPA), produzidas entre os anos de 2000 a 2020. Envolveu as atividades básicas de identificação, compilação, fichamento, análise e interpretação (adaptado de Marconi \& Lakatos, 2012).

A base utilizada para a coleta de dados foram: PubMed, SciELO, Google Acadêmico e livros físicos. Os termos de busca utilizados foram: Sistemas integrados, eficiência econômica de sistemas de produção, componente animal, componente lavoura, componente floresta, recuperação de pastagem, manejo de pastejo, sistemas de produção animal, desempenho produtivo e reprodutivo, estresse térmico em animais e efeitos fisiológicos de diferentes sistemas.

A análise dos dados foi realizada seguindo as perspectivas da técnica de Análise de Conteúdo (Bardin, 2010). Para a organização das informações, contidas nas publicações encontradas, foi realizado uma leitura flutuante e seleção dos trabalhos, para em seguida, identificação dos eixos temáticos e estabelecimento dos núcleos de sentido, resultando em 59 produções, composto por livros, artigos e literatura cinzenta (teses, dissertações, trabalhos apresentados em congressos, relatórios, etc.).

\section{Resultados e Discussão}

\subsection{Sistemas integrados de produção: desempenho, sustentabilidade e resiliência}

A vasta dimensão dos problemas e impactos ambientais atuais, associados a uma maior preocupação da sociedade com o meio ambiente e com a produção de alimentos, promoveu um novo cenário na cadeia de produção. Exigindo sistemas que garantam a produção e segurança alimentar sem agredir o ambiente. Diante da atual situação, vem se mostrando promissora a inserção da integração de sistemas de produção agropecuária, com maior ênfase no contexto de produção e sustentabilidade (Silva et al., 2016; Gasparini et al., 2017; Moraes et al., 2018).

Devido os sistemas integrados de produção agropecuária serem eficientes no uso dos recursos naturais, foram reconhecidos como alternativa para intensificação sustentável da produção. Os quais promovem boa taxa de ciclagem de nutrientes e melhoria do solo, reduzem os custos de produção e mantem níveis de produtividade elevados, além dos inúmeros serviços ecossistêmicos. Para tanto, são projetados para criar e aumentar as sinergias surgidas nas áreas de atividades integradas, entre pecuária, agricultura e silvicultura (Carvalho et al., 2014).

Essa integração entre os componentes dos sistemas integrados (solo, árvores, lavouras e animais), requer um manejo desafiador e diferente dos sistemas de monocultivo. Dos quais resultarão em efeitos positivos, negativos ou neutros. Esses efeitos poderão ainda, devido à dinâmica do sistema, oscilar em relação ao tempo e espaço, caracterizando a complexidade do sistema de integração (Carvalho et al., 2018b).

Podemos considerar que neste tipo de sistema, o principal compartimento é o solo, pois ele colhe e media os diversos processos sinérgicos das atividades, constituindo a "memória física". Já em relação aos nutrientes circulantes, de forma geral, o componente vegetal atua como acoplador de nutrientes e energia e o componente animal atua como catalisador e introdutor de novos fluxos de nutrientes (Carvalho et al., 2018a). 
Segundo Carvalho et al. (2018b), devido as possíveis diferentes escalas temporais e espaciais de interação, o componente animal pode interagir de duas formas dentro do sistema integrado. De forma direta e imediata, pelo pastejo, deposição de excreta e pisoteio, ou por interação mediata, pelo resíduo de esterco da fase de pastejo. Sendo então, o papel do animal reciclar e aumentar a eficiência do uso dos recursos (Anghinoni et al., 2013).

\subsection{Integrando o componente animal}

O componente animal beneficia o sistema integrado através do estímulo à produção de biomassa, aumento da ciclagem de nutrientes, aumento da massa e diversidade microbiana, melhoria nas condições físicas, químicas e da fertilidade do solo (Soares et al., 2018). A inclusão da pastagem com o componente animal aos sistemas pode gerar efeitos positivos ou negativos, dependendo da intensidade de pastejo (Vilela et al., 2011). $\mathrm{O}$ ato de colheita da parte área da forragem exercida pelo pastejo moderado provoca mudanças na estrutura do dossel da planta, resultando em maior estimulação da produção de biomassa (Silva, 2017). Com a deposição de fezes e urinas dos animais, ocorre a reciclagem de grande parte dos nutrientes. Nestas mesmas áreas sob dejetos, se observa aumento da matéria orgânica do solo (MOS), aumento dos íons trocáveis e do pH (Soares et al., 2018), além de fatores que indiretamente influenciam de forma positiva na biomassa e na diversidade microbiana (Chavez et al., 2011).

É importante ressaltar que estes fatores benéficos da inclusão do componente animal só ocorrem sob adoção correta da tecnologia de manejo/intensidade de pastejo e das mais adequadas, para as condições da área, caso contrário o efeito pode ser negativo, ocasionando perdas expressivas no sistema (Vilela et al., 2011). Neste sentido, um dos entraves para a inclusão do componente animal é o fato da possível compactação, causada pelo pisoteio. No entanto, Carvalho et al. (2018b) observaram que em sistemas integrados, a compactação pelo pisoteio animal, somente ocorre em altas intensidades de pastejo e nas camadas superficiais do solo (0-5cm), o que é passível de reversão com a lavoura (soja) de sucessão. Portanto, os efeitos de compactação teriam efeitos limitados e de vida curta.

Os sistemas integrados de produção agropecuária podem promover benefícios diretos e indiretos para a ordem zootécnica e ambiental. Os mais notórios benefícios são em relação à qualidade das forrageiras, devido à maior disponibilidade de nutrientes no solo e/ou pelo efeito positivo do sombreamento e na melhoria do conforto térmico, que influencia no bem estar animal e consequentemente em seu ganho de peso (Alves, 2012). Porém, cada modalidade/arranjo possui seus benefícios particulares e dependentes do correto manejo.

Outro fator benéfico dos sistemas integrados para a pecuária, seria a sua utilização em métodos alternativos na recuperação e renovação de pastagens em áreas degradadas. Sendo que as pastagens degradadas, tem sido atualmente, um dos fatores que mais vem comprometendo a sustentabilidade da produção animal (Macedo \& Araújo, 2012). No caso da integração lavoura pecuária (ILP), os benefícios iniciam no solo e na forragem, pois estes se beneficiam das adubações residuais das culturas anuais, refletindo em uma alta produção de forragem, e consequentemente influencia no desempenho animal (Balbinot et al., 2009; Arranha et al., 2019).

Paim et al. (2018) constataram que sistemas de ILP de alto insumo, produzem forragem de qualidade superior aos sistemas exclusivos de pecuária, podendo refletir em maiores ganhos de peso para os animais dos sistemas integrados. Segundo Alves et al. (2019) a temperatura do ar e a radiação solar são os fatores ambientais que mais influenciam no conforto animal, principalmente dos sistemas a pasto. Com a introdução do componente arbóreo em sistema com pecuária, consegue-se amenizar esses problemas, pois as árvores atuam modulando o microclima, através da formação de barreira física, reduzindo a incidência da radiação solar direta e da transpiração, reduzindo a temperatura do ar e elevando a umidade relativa (Arranha et al., 2019). 
Essas modificações ambientais são capazes de abrandar o estresse térmico sofrido pelo animal, favorecer o controle homeotérmico. Por conseguinte, podem melhorar o desempenho produtivo, reprodutivo e sanitário do animal (Nicodemo et al., 2018; Alves, 2012), além dos ganhos "intangíveis". Vale ainda lembrar que por mais que esteja diretamente ligada, ambiência não é sinônimo de bem estar animal (Alves et al., 2019).

Segundo Nicodemo et al. (2018), o componente arbóreo também influencia na questão alimentar dos animais, devido a possíveis efeitos positivos na qualidade nutricional da forrageira e na manutenção da fertilidade do solo, o que reflete na produção de forragem. Além de promover um enriquecimento ambiental, atuando positivamente no comportamento dos animais. O efeito dos sombreamentos das árvores pode influenciar em aumento, redução ou ausência sobre algumas características de produção e qualidade das forrageiras, dependendo da espécie forrageira, época do ano e percentagem de sombreamento. Portanto, em sistema integrado com o componente arbóreo tem-se a possibilidade de aumento do valor nutritivo da forragem, o qual está muito ligado a escolha e manejo da espécie, tanto forrageira quanto arbórea (Paciullo et al., 2009).

Paciullo et al. (2007) verificaram maiores teores de proteína bruta, redução de fibra em detergente neutro e incremento da digestibilidade in vitro da matéria seca da Brachiaria decumbens, entretanto, não constataram redução nos valores de massa de forragem, densidade de perfilhos e índice de área foliar da B. decumbens em sombreamento moderado (35\% de sombra), diferente do observado em sombreamento intenso (65\% de sombra), em que foi constatada a redução. Este fato reforça a necessidade de escolha correta das espécies e do manejo, para efeitos positivos da integração.

O consumo de forragem e o desempenho animal, em sistemas a pasto, dependem do valor nutritivo e da disponibilidade da forragem. Logo, sistemas sombreados que influenciam positivamente na qualidade das forragens devem ser considerados, pois em tese refletem em maiores ganhos de produção animal (Almeida et al., 2019). Portanto, é imprescindível que para a integração do componente arbóreo ser benéfica ao componente pecuário, ele tenha uma copa que proporcione boa área de sombreamento para os animais, mas que também permita em quantidade suficiente a passagem de radiação solar para o desenvolvimento forrageiro (Alves et al., 2019).

\subsection{Manejo do componente animal: o ponto para o sucesso da integração}

\subsubsection{Estresse térmico nos animais}

Os animais possuem uma íntima relação com o ambiente em que são criados. Portanto, as condições do ambiente, principalmente às condições ambientais climáticas, influenciam diretamente no conforto e bem estar animal. Assim, a interação animal e ambiente deve ser considerada quando se busca maior produtividade (Costa et al., 2012).

A zona de conforto térmico, também chamada de zona termoneutra, é uma faixa de temperatura ambiente da qual o animal se encontra em equilíbrio e conforto para suas manutenções fisiológicas, ou seja, em homeostase, sem a utilização de mecanismos termorreguladores. Nesta faixa de temperatura ideal, consideramos que o animal se encontra em condições perfeitas para expressar suas melhores características produtivas (Silva et al., 2020). No entanto, quando as temperaturas do ambiente estão em níveis críticos, fora da faixa de termoneutralidade, o animal responde com reações fisiológicas e comportamentais para tentar se adaptar as novas condições, principalmente através da variação da temperatura corporal e utilização de energia para termorregulação (Nicodemo et al., 2018).

Esse desequilíbrio na temperatura pode provocar ao animal um estresse por frio (hipotermia), quando ocorre uma temperatura crítica inferior, ou estresse por calor (hipertermia), quando ocorre uma temperatura crítica superior, sendo a mais comum. Quando um animal sofre estresse por calor, devido às temperaturas críticas superiores a zona de conforto térmico, é desencadeado um efeito sistêmico e a ativação de mecanismos de termorregulação. Como a homeotermia é prioridade para os 
animais, eles reduzem as atividades que produzem calor e aumentam atividades que promovem perda de calor (Nicodemo et al., 2018; Silva et al., 2020).

Conforme a intensidade e duração do estresse ocorrem efeitos sistêmicos como aumento respiratório, aumento no fluxo sanguíneo para órgãos de funções primordiais (coração, pulmão e cérebro), inibição a ingestão de alimento e a digestão, inibição da imunidade e inibição funções reprodutivas (Burdick et al., 2011). Dependendo do grau desses efeitos, tanto a saúde quanto as funções são prejudicadas. Os parâmetros como crescimento e desenvolvimento, reprodução e lactação podem apresentar quedas, com prejuízos ao processo produtivo (Façanha et al., 2013).

A zona de conforto está atrelada a fatores relativos ao ambiente, como temperatura e umidade relativa do ar, radiação solar, velocidade do vento e também a fatores próprios do animal como a espécie, idade, característica física, nível/estado reprodutivo, nível de produção e nível de alimentação (Nicodemos et al., 2018). Animais mais produtivos tendem a produzir mais calor e serem mais sensíveis a temperaturas altas, logo o aumento na produtividade, em grande maioria, afeta a faixa de zona de conforto. Um ótimo exemplo são as vacas leiteiras, das quais os genótipos mais produtivos são menos adaptados às condições climáticas de alta temperatura, e quando impostas a essas condições, apresentam marcante queda na ingestão de alimento e consequentemente na produção de leite (Façanha et al., 2013).

As diferentes zonas de conforto e adaptação a determinadas faixas de temperatura variam de acordo com as espécies. Este fato pode ser observado com as constatações da pesquisa de Paim et al. (2018), que ao avaliarem novilhas nelores puras e F1 (Nelore $\times$ Angus) sob estresse térmico de clima tropical, observaram que as nelores apresentam menores temperaturas termográficas, possivelmente devido a menor produção de calor para as manutenções fisiológicas e melhor termorregulação. Sendo assim, as novilhas nelores aparentaram estar mais adaptadas ao estresse térmico de clima tropical que as F1 (Nelore $\times$ Angus). No entanto, mesmo os animais considerados bem adaptados a climas quentes, podem sofrer um período de estresse e terem perdas mínimas de produção (Façanha et al., 2013).

Os fatores climáticos impõem a necessidade de termorregulação aos animais, o que pode resultar em redução do consumo voluntário e do desempenho dos mesmos. Galloso-Hernández et al. (2020), observaram que em sistemas convencionais com estresse térmico intenso, os animais reduziram o tempo de pastejo (apesar da disponibilidade), subentendendo que para os animais, em níveis intensos de estresse térmico, a defesa do calor é mais importante que a alimentação. O desafio ambiental é ainda maior para os animais submetidos ao estresse térmico no período seco/estação seca, os quais passam por maior dificuldade em se adaptar, resultando em um maior impacto no desempenho (Paim et al., 2018).

Em vacas lactantes, o efeito negativo de altas temperaturas é perceptível no comportamento, ingestão de alimento e desempenho produtivo. Vacas leiteiras de alta produção tendem a sofrer maior estresse térmico, pois geram mais calor em relação às de baixa produção e não o dissipam de modo eficiente, podendo apresentar quedas em torno de $22 \%$ da produção de leite em vacas de $40 \mathrm{~kg} /$ dia e $17 \%$ da produção em vacas de $15 \mathrm{~kg}$ de leite/dia. Além da queda na produção de leite, ocorre alteração na composição do leite com redução nos teores de gordura, proteína, cálcio, lactose, ácido cítrico e potássio (Melo et al., 2016).

Levando em consideração a sombra e o microclima promovido pelo componente arbóreo em sistemas de integração, estes têm o potencial de diminuir o estresse térmico em animais e melhorar o seu bem estar. Em búfalos mantidos em diferentes ambientes, foi constatada diminuição do chafurdamento associado a um aumento significativo do tempo gasto no sombreamento pelos animais (Galloso-Hernández et al., 2020). Dados similares foram constatados por Schutz et al. (2011), que ao avaliarem a preferência de vacas leiteiras por sombra ou condições ambientais, no período de fevereiro a março (verão no hemisfério sul) com temperatura média do ar de $22,3^{\circ} \mathrm{C}$, notaram que a preferência dos animais pela sombra em relação às condições ambientais aumentou com a temperatura do ar (aumento de $12 \%$ por aumento de $1^{\circ} \mathrm{C}$ na temperatura), radiação solar (aumento de $8 \%$ por $100 \mathrm{~W} / \mathrm{m}^{2}$ ) e velocidade do vento (aumento de $4 \%$ por aumento de $1 \mathrm{~km} / \mathrm{h}$ ). Algumas medidas que 
auxiliam na convivência do animal com elevadas temperaturas são recomendadas: provisão de sombra, aspersão de água e o fornecimento de dieta rica em nutrientes (Nicodemo et al., 2018).

Além de prejudicar o desempenho produtivo dos animais, o estresse por calor também interfere na reprodução de machos e de fêmeas, através do comprometimento das funções reprodutivas, geradas pelas alterações na homeostase. $\mathrm{Na}$ tentativa de perder mais calor sensível, pelo redirecionamento de sangue para a periferia do corpo e na redução na produção de calor metabólico, pela redução do consumo de ração, ocorre alterações fisiológicas, no balanço energético e na disponibilidade de nutrientes, que podem indiretamente comprometerem a ciclicidade, o estabelecimento de gestação e o desenvolvimento fetal (Costa et al., 2016).

Nas fêmeas o estresse por calor pode afetar todas as etapas (iniciais até maturação) do desenvolvimento dos folículos e qualidade dos oócitos. Pode atrasar ou inibir o desenvolvimento embrionário, interferir no número e qualidade de embriões in vitro, reduzir a taxa de gestação e desencadear distúrbios gestacionais (Silva et al., 2020). Ademais, em fêmeas sob estresse térmico pode ser observada uma menor duração e intensidade do estro, aumento na incidência de cio silencioso e do anestro, devido principalmente a uma redução nos níveis de estradiol, o que resulta em queda no número de inseminações e de prenhes/inseminação (Costa et al., 2016).

Essa redução da concentração de estradiol associada às alterações nas secreções de hormônio luteinizante, hormônio folículo estimulante e inibina, podem afetar o desenvolvimento folicular pela diminuição na dominância folicular e no tamanho dos folículos dominantes. Ocorre também, diminuição da esteroidogênese folicular. A qualidade do oócito pode ser prejudicada pelo estresse térmico, principalmente durante o período pré ovulatório, o que está muito associado às alterações hormonais. Todas essas mudanças levam a alterações e redução na eficiência do ovário (Rocha et al., 2012).

A elevada temperatura que um embrião for exposto pode gerar alterações no seu desenvolvimento e até a morte. Quando a fêmea passa por estresse no dia da concepção e/ou até sete dias após concepção, pode ocorrer maiores índices nas falhas de desenvolvimento e menores taxas de sobrevivência embrionária (Costa et al., 2015; Fialho et al., 2018). Já quando sofrido pela fêmea durante a gestação, leva a uma redistribuição do sangue para a periferia corpórea, ocasiona redução da perfusão placentária, podendo causar efeitos negativos no crescimento fetal, nos pesos placentários e nas concentrações de hormônios placentários circulantes (Costa et al., 2016).

Oliveira et al. (2013) ao avaliarem as possibilidades de declínio na taxa de concepção de vacas leiteiras em relação a temperatura do ambiente e estresse térmico, constataram queda de 30\% a 35\% na taxa de concepção de vacas lactantes durante os meses mais quentes do ano, com tendência a queda drástica no início do período mais quente e de maior estresse e gradativa recuperação com o final do mesmo. Neste sentido, foi avaliado o impacto da oferta de sombra em sistemas de integração lavoura pecuária (ILP) e integração lavoura pecuária floresta (ILPF) sob desenvolvimento embrionário in vitro e parâmetros fisiológicos relacionados à resposta ao estresse em novilhas nelores (Bos indicus) e se observou que novilhas nelores manejadas em ILPF apresentaram maior produção de embriões in vitro, sem alterações típicas em seus parâmetros fisiológicos (Silva et al., 2020).

Nos machos, os principais efeitos deletérios reprodutivos desencadeados pelo estresse térmico, estão associados a redução na taxa de crescimento corporal e testicular, o que também está relacionado com a disponibilidade nutricional (Costa et al., 2015). Nicodemo et al. (2018) afirmam que em machos sob estresse térmico são observados principalmente redução da produção e motilidade de espermatozoides, alteração da concentração de testosterona e ejaculado com maior número de espermatozoides morfologicamente anormais. As alterações mais intensas, observadas nos machos sob estresse calórico são nas espermatogônias, espermatócitos e espermátides.

Devido os efeitos deletérios em todas as fases da reprodução e redução da eficiência reprodutiva dos animais, desencadeado pelo estresse térmico, se faz necessário a adoção de medidas que reduzam esses impactos. Medidas que vão 
desde seleção de animais mais tolerantes ao calor, até à modificação do ambiente para prover maior conforto térmico aos animais (Macedo et al., 2013). Uma boa opção de modificação de ambiente é o emprego de sistemas integrados com o componente arbóreo, pois o sombreamento reduz a carga de calor radiante nos animais e consequentemente reduz o calor corporal e facilita a termorregulação, favorecendo o bem-estar animal e a produtividade (Silva et al., 2020).

\subsubsection{Manejo da pastagem}

O manejo da pastagem e do pastejo, são práticas muito importantes para o sucesso dos sistemas de integração, portanto após o estabelecimento da pastagem no sistema, a duração do período de pastejo, a intensidade de pastejo e a adubação estão entre os principais fatores de manejo (Soares et al., 2018). A produção de forragem em sistemas integrados pode ser influenciada por diversos fatores como clima, práticas de manejo e dinâmica de competição acima e abaixo do nível do solo. Devido a isso, as espécies/cultivares escolhidas para a implantação em sistemas integrados devem apresentar características de boa produção e tolerância a sombreamento (Almeida et al., 2019).

Segundo Ferreira (2020), para sistemas de integração, as gramíneas consideradas tolerantes ao sombreamento e com produção de forragem satisfatória são Brachiaria brizantha cvs. Marandu, Xaraés e Piatã, Brachiaria decumbens cv. Basilisk, Panicum maximum cvs. Aruana, Mombaça e Tanzânia e Panicum spp. cv. Massai. Já as forrageiras leguminosas geralmente são menos tolerantes ao sombreamento do que as gramíneas. As calopogônio (Calopogonium mucunoides), centrosema (Centrosema pubescens) e puerária ou kudzu-tropical (Pueraria phaseoloides) são consideradas medianamente tolerantes ao sombreamento, já a Arachis pintoi apresenta boa tolerância ao sombreamento, porém possui um processo de estabelecimento mais demorado (Almeida et al., 2019).

Para evitar problemas de competição entre as espécies e possível inviabilização do cultivo consorciado, o conhecimento do comportamento das espécies na competição por fatores de produção também é de extrema importância para o sucesso da integração (Paris et al, 2009). Outro ponto importante, nos sistemas integrados é a duração do período de pastejo, o qual é dependente do período das culturas em consórcio. Considerando as espécies forrageiras e culturas, as operações de semeadura e colheita e o tempo de diferimento, o restante seria para a produção animal. Portanto, quanto mais tempo o animal estiver sobre a pastagem, maior a oportunidade de otimizar a produção animal (Moraes et al., 2018). Soares et al. (2018), consideram possíveis a diminuição ou exclusão de algumas janelas entre o cultivo por meio de algumas ações: semear o pasto assim que a colheita de grãos for realizada ou, se possível, antes da própria colheita; manter o pasto a um nível de massa de forragem adequado para a formação de palhada, excluindo a necessidade de retirada dos animais dias antes da dessecação; dessecar o pasto o mais próximo possível da semeadura; elaborar misturas forrageiras com ciclos complementares aumentando o período de pastejo, antecipando com espécies/cultivares precoces e retardando a saída com espécies tardias; fertilização do pasto de forma escalonada; e considerar escalonamento de semeadura e colheita.

A criação de animais com base alimentar em pastagem se baseia no consumo de forragem e no desempenho animal, fatores que são influenciados pelo manejo das pastagens, refletindo no seu valor nutritivo e disponibilidade (Almeida et al., 2019). O manejo dos animais sobre o pasto consiste em estabelecer a taxa de lotação em relação a intensidade de pastejo e estabelecer o método de lotação/distribuição dos animais na área. Estudos realizados em sistemas integrados têm demonstrado que o método de lotação não é uma ferramenta decisiva para a produtividade do sistema, mas sim a intensidade de pastejo que realmente define se um sistema de integração terá sucesso, produtividade e sustentabilidade (Carvalho et al., 2018a). Vale ressaltar que áreas de pastagem exclusivas para a produção de palhada que não sofrem pastejo, de plantio direto em sucessão, não produzem mais do que áreas pastejadas (Carvalho et al., 2018b).

A intensidade de pastejo caracteriza-se pela relação de animais no pasto e a massa de forragem da área, refletindo então a pressão do pastejo e as consequências do componente animal sob a área. Portanto, é considerada um dos manejos 
capazes de levar a grandes consequências (positivas e negativas) (Soares et al., 2018). Considerando a influência sobre o desempenho animal, tanto per capita quanto por área, e na determinação da quantidade e a qualidade da biomassa residual remanescente na superfície do solo após o período de estocagem para a cultura subsequente, a intensidade de pastejo é essencial para promover feedbacks positivos dentro do sistema (Carvalho et al., 2018b).

É necessário um criterioso manejo de forragem, evitando ao máximo manter a altura de pastejo abaixo do recomendado para a espécie/cultivar forrageira, possibilitando maior acúmulo de reservas e favorecendo a rebrotação (Almeida et al., 2019). Carvalho et al. (2018b) compilaram dados sobre intensidades de pastejo em diferentes sistemas integrados, dos quais evidenciam efeitos negativos de altas intensidades de pastejo, ocasionado em todo o sistema. Já em intensidade de pastejo leve e moderada, evidenciaram diversos favorecimentos para a resiliência da atividade.

O sucesso das atividades que envolvem a pecuária a pasto, tem como ponto chave o manejo de pastagem. Principalmente para os sistemas integrados, devido ao componente lavoura poder ser afetado e aos efeitos do manejo (positivos ou negativos) serem potencializados (Soares et al., 2018). Portanto a intensidade de pastejo leve a moderada, tem proporcionado os melhores efeitos positivos dentro de sistemas integrados com componente animal (Carvalho et al., 2018b).

\subsubsection{Tipo animal (ênfase em bovinos)}

A escolha do tipo animal deve ser baseada em cadeias produtivas estruturadas. O Brasil possui uma predominância na utilização de ruminantes nos sistemas integrados, geralmente representada por bovinos de corte, mas também ocorre a utilização de bovinos de leite e pequenos ruminantes (ovinos e caprinos) (Soares et al., 2018). Vale ressaltar que o sistema de produção pecuário, especificamente a bovinocultura, é o conjunto de tecnologias e práticas de manejo, bem como o tipo de

animal, o propósito e modelo da criação e a raça. É caracterizado como um sistema complexo e diversificado, não havendo fórmulas e nem recomendações únicas que possam ser largamente aplicadas. Neste sentido, há a necessidade de desenvolver cada sistema de produção de acordo com as metas, com as condições de ambiente e de mercado (Abreu et al., 2003; Santos, 2011).

\subsubsection{Bovino de corte}

O rebanho brasileiro é composto principalmente por animais zebuínos, com destaque à raça Nelore e seus mestiços, possivelmente devido suas boas características de adaptação ao ambiente. Raças zebuínas (Bos taurus indicus) apresentam maior rusticidade, boa adaptação às condições climáticas tropicais e resistência a endo e ectoparasitas, em função de seus atributos anatomofisiológicos, porém, principalmente, em rebanhos não selecionados apresentam menor ímpeto de crescimento e carcaças com menor uniformização as raças europeias (Bos tauros taurus), que, por sua vez para expressarem esse potencial são mais exigentes em condições ambientais favoráveis. O cruzamento entre raças zebuínas e europeias visa à exploração da heterose e da complementaridade entre as raças. Com isso, os produtos oriundos do cruzamento tendem a apresentar maior precocidade, melhor adaptabilidade e resistência (Barbosa et al., 2014; Costa, 2017).

O uso de cruzamentos vem ganhando importância no cenário atual, pelos melhores desempenhos observados nas características de importância econômica, como, características de carcaça e carne, bem como características reprodutivas (Costa, 2017). Frente às atuais exigências de intensificação sustentável na pecuária, devemos utilizar, quando cabíveis ao sistema, os recursos genéticos de alto rendimento, principalmente os de cruzamento. No Brasil um dos cruzamentos mais utilizados é de zebuínos com raças selecionadas para carne, em destaque o de Nelore (fêmea) e Aberdeen Angus (Pain et al., 2018). Barbosa et al. (2014) compararam o desempenho de animais da raça Tabapuã e de cruzamentos de diferentes grupos genéticos (1/2 Red Angus x 1/2 Tabapuã; 1/2 Santa Gertrudes x 1/4 Red Angus x 1/4 Tabapuã e 1/2 Santa Gertrudes x 1/2 Tabapuã) recriados e terminados a pasto. Observaram que os animais cruzados apresentaram melhor desempenho que os puros, com 27 
$\mathrm{kg}$ a mais no peso a desmama e abatidos mais cedo. No desempenho final os animais $1 / 2$ Red Angus $x 1 / 2$ Tabapuã apresentaram desempenho superior aos animais puros e foram abatidos mais pesado, os outros cruzamentos não apresentaram diferenças no desempenho final da raça pura.

Animais de alto potencial genético são indicados para sistemas de integração, para alcançar elevados ganho de peso, obter maiores rendimento de carcaça e precocidade de abate (Fraser et al., 2014). Costa (2017) corrobora que a utilização de animais cruzados de corte em sistemas intensivos, incluindo em sistemas integrados, favorece o abate precoce. Neste contexto, cresce a utilização de animais precoces e superprecoces, o que tem sido responsável por melhorias nas ofertas de produtos de boa qualidade (Euclides et al., 2003). Vale ressaltar que a melhoria no bem estar animal proporcionada pelo componente arbóreo em alguns sistemas de integração, possibilita a adição da genética de raças europeias e britânicas ao rebanho do Brasil tropical, o que pode ser aproveitado, para melhorar a qualidade da carne produzida e alçar novos mercados (Soares et al., 2018). Em momentos de baixa do preço de boi gordo, ocorre um aumento considerável de abate de fêmeas, realizados como "estratégias" pelos produtores. As fêmeas podem ser consideradas uma importante fonte de produção de carne, desde vacas de descarte a novilhas de abate (Santos et al., 2008).

Segundo Silva (2017), o cruzamento industrial tem sido um dos fatores que tem colaborado para o aumento de fêmeas no abate, sobretudo na região Centro-Oeste do Brasil. Por serem menores do que os machos e se desenvolvem mais lentamente, no entanto, mais precoces em acabamento de carcaça, as fêmeas apresentam potencial para sistemas que buscam o abate de animais jovens, bem terminados e com carne de qualidade (Euclides, 2000). Um dos grandes benefícios para a utilização de fêmeas para abate seria em situações de sistemas em consórcio, sucessão e rotação de cultura que resultam em menor entressafra, ocasiões onde o menor período de terminação para as fêmeas em relação os machos seriam o mais indicado ao sistema (Soares et al., 2018). De forma geral o objetivo produtivo na bovinocultura de corte é produzir a maior quantidade de quilos de carne por área, de forma rentável. Para tanto, cada uma dessas fases mencionadas possui distintos objetivos. Como a fase de cria é o ciclo responsável pela produção e venda de bezerros, seu objetivo principal é o desmame de bezerro/ano/matriz, para isso busca-se obter o maior número de bezerros por vaca acasalada (taxa de natalidade) e o maior peso a desmama possível (Barbosa et al., 2015).

Segundo Soares et al. (2018) a eficiência reprodutiva desta categoria está em estreita relação com o peso e condição da matriz, o que é determinante para atingirem adequadas taxas de prenhez e natalidade, que são os índices de maior impacto na produtividade, assumindo maior importância que o ganho de peso dos bezerros. Abreu et al. (2003) corroboram que a fertilidade das matrizes é o componente de maior impacto econômico na produção de bovinos de corte na fase de cria. Portanto o manejo reprodutivo é a chave da produtividade e do desempenho do rebanho.

Como formas para solucionar e/ou amenizar o efeito negativo do estado nutricional das matrizes no período de estação de monta, é aconselhável o investimento em nutrição e suplementação das mesmas, principalmente nos períodos de condições adversas, outono/inverno (na região sul) e período seco (região tropical) (Nogueira et al., 2015). De acordo com Soares et al. (2018), produtores do sul do Brasil vem utilizando estratégias na fase de cria, para melhorar o estado nutricional das matrizes. Estão antecipando a estação de monta (Ago-Nov), o que leva a parição sobre as pastagens de inverno (jul-set), normalmente em sistemas integrados, possibilitando que o melhor nível nutricional das vacas ocorra no pós-parto, trazendo melhores resultados nos índices reprodutivos.

No Brasil, a fase de recria é a que retém por maior período os animais, especialmente nos sistemas tradicionais de produção. Nas fases de recria e engorda da bovinocultura de corte, o controle zootécnico deve ser focado no desempenho produtivo da unidade de produção, que são os novilhos(as). Neste caso, o principal indicador de desempenho é o ganho de peso vivo (diário - g/dia e/ou anual- $\mathrm{kg} / \mathrm{ano}$ ), que influencia diretamente na idade de abate dos animais. Já para a produtividade, o principal indicador é a quantidade de peso vivo produzida anualmente em cada hectare de pastagem ( $\mathrm{kg} / \mathrm{ha} / \mathrm{ano}$ ) (Andrade et 
al., 2005). Muitos produtores estão investindo em animais de alto potencial genético para alcançar elevado ganho de peso, obter maior rendimento de carcaça e precocidade de abate (Fraser et al.,2014). Estes esforços resultam em maior produção e abate de novilhos precoces (24-26 meses de idade e 480-540 kg de peso vivo) e superprecoces (13-15 meses de idade e 440$500 \mathrm{~kg}$ de peso vivo) (Nogueira et al., 2015), otimizando áreas e trazendo melhores rendimentos.

Em sistemas de criação de bovinos em pastagens, o manejo da pastagem exerce forte influência tanto no desempenho quanto na produtividade animal (Andrade et al., 2005). Nogueira et al. (2015) consideram que quanto maior for o peso de desmama do animal que entra na fase de recria, menor será o tempo da recria, consequentemente menor será o período de abate, além de maior possibilidade de entrada de fêmeas na fase reprodutiva. Nesta linha, Frizzo et al. (2003) visam o estudo e desenvolvimento de sistemas integrados viáveis de alimentação e manejo na recria de bezerras como ferramenta para acelerar o crescimento de fêmeas no período pós-desmama e reduzir a idade de acasalamento. Para Soares et al. (2018), a utilização de sistemas integrados associada a suplementação é uma alternativa viável para a terminação de bovinos. O confinamento estratégico ao final do período de pastagem também é uma alternativa que pode garantir o acabamento dos animais. Portanto, a utilização de tecnologias que otimizem o desempenho animal é fundamental para a bovinocultura de corte alcançar produção de forma sustentável e competitiva (Figueiredo et al., 2007).

\subsubsection{Bovinos de leite}

A bovinocultura leiteira no Brasil é fonte de renda para milhares de produtores e fornecedora de um dos principais insumos da indústria alimentícia. Nacionalmente, apresenta como caraterística uma grande diversidade produtiva e de níveis tecnológicos (Araújo et al., 2013). Dentre os recursos genéticos para produção de leite estão as raças europeias provenientes de Bos taurus taurus e indianas do grupo Bos taurus indicus, além dos diversos grupos genéticos obtidos a partir de cruzamentos, inclusive raças sintéticas e compostas (Ximenes \& Martins, 2018). De modo geral, no Brasil predominam as raças Holandesas, Jersey, Gir leiteiro e seus cruzamentos (Soares et al., 2018).

Nesta gama de opções, a escolha deve ser pelo genótipo que melhor se enquadre dentro dos padrões do sistema de produção, sendo um dos maiores entraves na decisão. Sobretudo, os mais produtivos, são mais sensíveis ao estresse térmico e respondem rapidamente com quedas significativas no consumo de alimentos e na produção. Devido a isso, uma alternativa muito utilizada é a associação de genótipos produtivos a animais adaptados ao clima (Façanha et al., 2013).

O principal problema que restringe a rentabilidade dos sistemas leiteiros nacionais, de forma geral, é a baixa produtividade das fêmeas, decursivo principalmente do ineficiente manejo alimentar (Cunha et al., 2018). Outro entrave para a produção, mais específico para as regiões tropicais do país, são os problemas oriundos da utilização de genótipos não adaptados ao clima, sem a adoção das tecnologias necessárias para a amenização das condições climáticas aos animais (Façanha et al., 2013). Bovinos leiteiros, principalmente os mais produtivos, apresentam uma considerável produção de calor metabólico, o que dificulta o seu equilíbrio térmico, quando submetidos a condições de calor, sendo mais susceptíveis ao estresse térmico (Carnevalli et al., 2019). Melo et al. (2016) mencionam diminuição de produção de leite em vacas sob estresse calórico, em torno de $17 \%$ a $22 \%$ e alteração na composição do leite com redução nos teores de gordura, proteína, cálcio, lactose, ácido cítrico e potássio. Neste aspecto os sistemas integrados entram como uma boa opção para a integração da bovinocultura leiteira, dependendo do arranjo, podem fornecer forragem de boa qualidade, sombreamento e microclima mais favoráveis para a categoria (Santos et al., 2013).

\section{Conclusão}

Com o intuito de discutir a integração e os benefícios do componente animal em sistemas integrados de produção agropecuária, conclui-se que os sistemas integrados de produção agropecuária são projetados para criar e aumentar as sinergias 
surgidas nas atividades integradas. A introdução do componente pastoril pode aumentar a intensificação sustentável destes sistemas, porém os deixam mais complexos. Nestes casos, a intensidade de pastejo é a ferramenta que realmente conduz o funcionamento do sistema, sendo a intensidade moderada de pastejo a mais recomendada para conciliar produtividade e sustentabilidade ao sistema.

Já os benefícios em sistemas integrados de produção agropecuária que contêm no seu arranjo o componente animal, ocorre como vias de mão duplas. O componente animal pode promover benefícios diretos e indiretos para os outros componentes do sistema, seja pelo pastejo, deposição de excreta e pisoteio. Diante disso, a função geral do componente animal dentro de sistemas integrados é reciclar e aumentar a eficiência do uso dos recursos. Os demais componentes dos sistemas também podem promover benefícios diretos e indiretos para o componente animal, sendo os mais notórios a qualidade das forrageiras e a melhoria no conforto térmico e bem estar animal. O estresse térmico nos animais pode gerar inúmeras alterações fisiológicas, afetando negativamente o bem estar, a produção e reprodução.

\section{Referências}

Abreu, U. G. P., Cezar, I. M. \& Torres, R. A. (2003). Análise Bioeconômica da Introdução de Período de Monta em Sistemas de Produção de Rebanhos de Cria na Região do Brasil Central. Revista Brasileira de Zootecnia, 32(5), 1198-1206. http://doi.org/10.1590/S1516-35982003000500021.

Almeida, E. M., Almeida, R. G., Miyagi, E. S., Freitas, P. V. D. X., Ribeiro, F. M., Fernandes, P. B. \& Garcia, E. C. (2019). Sistemas silvipastoris: uma abordagem sobre a interação dos componentes bióticos e abióticos. Revista Científica Rural, 21. http://doi.org/10.30945/rcr-v21i2.353

Alves, F. V. (2012). O componente animal em sistemas de produção em integração. In: Bungenstab, D. J. (ed). Sistemas de integração lavoura-pecuáriafloresta: a produção sustentável. (2a. ed., pp. 143-154) Brasília, GO: Embrapa. https://www.embrapa.br/busca-de-publicacoes/-/publicacao/938814/sistemasde-integracao-lavoura-pecuaria-floresta-a-producao-sustentavel.

Alves, F. V. \& Karvatte Junior, N. (2019). Benefícios da sombra em sistemas em integração lavoura-pecuária-floresta nos trópicos. In: Bungenstab, D. J., Almeida, R. G., Laura, V. A., Balbino, L. C. \& Ferreira, A. D. ILPF: inovação com integração de lavoura, pecuária e floresta. (525-541). Brasília, GO: Embrapa. https://www.embrapa.br/busca-de-publicacoes/-/publicacao/1113064/ilpf-inovacao-com-integracao-de-lavoura-pecuaria-e-floresta.

Alves, F. V., Silva, V. P. \& Karvatte Junior, N. (2019). Bem-estar animal e ambiência na ILPF. In: Bungenstab, D. J., Almeida, R. G., Laura, V. A., Balbino, L. C. \& Ferreira, A. D. ILPF: inovação com integração de lavoura, pecuária e floresta. (pp. 207-224). Brasília: Embrapa. https://www.embrapa.br/busca-depublicacoes/-/publicacao/1113064/ilpf-inovacao-com-integracao-de-lavoura-pecuaria-e-floresta.

Andrade, C. M. S., Ferreira, J., Cavalcante, V. F. A. \& Valle, L. A. R. (2005). Padrões de desempenho e produtividade animal para a recria-engorda de bovinos de corte no Acre. Embrapa Acre. Rio Branco, AC. https://www.embrapa.br/busca-de-publicacoes/-/publicacao/505044/padroes-de-desempenho-eprodutividade-animal-para-recria-engorda-de-bovinos-de-corte-no-acre.

Anghinoni, I., Carvalho, P. C. F. \& Costa, S. E. V. G. A. (2013). Abordagem sistêmica do solo em sistemas integrados de produção agrícola e pecuária no subtrópico brasileiro. In: Araújo, A. P. \& Alves. B. J. R. Tópicos em Ciência do Solo - VIII. Sociedade Brasileira de Ciência do Solo (v. 5, p.325-380). https://www.researchgate.net/publication/260793648_Abordagem_sistemica_do_solo_em_sistemas_integrados_de_producao_agricola_e_pecuaria_no_subtro pico_brasileiro.

Aranha, H. S., Andrighetto, C., Lupatini, G. C., Bueno, L. G. F., Trivelin, G. A., Mateus, G.P., Luz, P. A. C., Santos, J. M. F., Sekiya, B. M. S. \& Vaz, R. F. (2019). Produção e conforto térmico de bovinos da raça Nelore terminados em sistemas integrados de produção agropecuária. Arquivo Brasileiro de Medicina Veterinária e Zootecnia. 71(5):1686-http://doi.org/10.1590/1678-4162-9913.

Araújo, A. P., Oliveira, V. J., Siqueira, J. V. M., Mousquer, C. J., Freiria, L. B., Silva, M. R., Ferreira, V. B., Silva Filho, A. S. \& Santos, C. M. S. (2013). Qualidade do leite na bovinocultura leiteira. Publicações em Medicina Veterinária e Zootecnia. 7(22). https://www.pubvet.com.br/artigo/787/qualidade-doleite-na-bovinocultura-leiteira.

Balbinot Junior, A. A., Moraes, A., Veiga, M., Pelissari, A. \& Dieckow, J. (2009). Integração lavoura-pecuária: intensificação de uso de áreas agrícolas. Ciência Rural. http://doi.org/ 10.1590/S0103-84782009005000107.

Barbosa, F. A., Borges, D. N., Cabral Filho, S.L.S., Graça, D. S., Andrade, V. J., Souza, C. E., Leão, J. M. \& Mandarino, R.A. (2014). Desempenho de bovinos Tabapuã e seus cruzados em pastagens de braquiária no estado da Bahia. Arquivo Brasileiro de Medicina Veterinária e Zootecnia, 66(1): 253-258. http://doi.org/10.1590/S0102-09352014000100034.

Barbosa, F. A., Soares-Filho, B. S., Merry, F. D., Azevedo, H. O., Costa, W. L., Coe, M. T., Batista, E. L. S., Maciel, T. G., Sheepers, L. C., Oliveira, A. R. \& Rodrigues, H. O. (2015). Cenários para a pecuária de corte amazônica. Belo Horizonte, MG: Instituto de Geociências. https://csr.ufmg.br/pecuaria/wpcontent/uploads/2015/03/relatorio_cenarios_para_pecuaria_corte_amazonica.pdf.

Bardin, L. (2010). Análise de conteúdo. (4a ed.). Edições70.

Burdick, N. C., Randel, R. D., Carroll, J. A. \& Welsh Junior, T. H. (2011). Interactions between Temperament, Stress, and Immune Function in Cattle. International Journal of Zoology. http://doi.org/10.1155/2011/373197. 
Carnevalli, R. A., Coleti, A. J., Tonini, H., Silva, A. B., Giustina, C. D. \& Lulu, J. (2019). Princípios da pecuária leiteira em sistemas de ILPF. In: Bungenstab, D. J., Almeida, R. G., Laura, V. A., Balbino, L. C. \& Ferreira, A. D. ILPF: inovação com integração de lavoura, pecuária e floresta. (1ed., p. 225-240). Brasília, GO: Embrapa. Disponível em: https://www.embrapa.br/busca-de-publicacoes/-/publicacao/1113064/ilpf-inovacao-com-integracao-de-lavourapecuaria-e-floresta.

Carvalho, C. F., Peterson, C. A., Nunes, P. A. A., Amanda, A. A., Martins, A. P., Souza Filho, W., Bertolazi, V. T., Kunrath, T. R., Moraes, A. \& Anghinoni, I. (2018a). Animal production and soil characteristics from integrated crop-livestock systems: toward sustainable intensification. American Society of Animal Science. pp. 3513-3525. http://doi.org/10.1093/jas/sky085.

Carvalho, P. C. F., Barro, R. S., Barth Neto, A., Nunes, P. A. A., Moraes, A., Anghinonis, I., Bredemeier, C., Bayer, C., Matins, A. P., Kunrath, T. R., Santos, D. T., Carmona, F. C., Barros, T., Souza Filho, W., Almeida, G. M., Caetano, L. A. M., Cecagno, D., Arnuti, F., Denardin, L. G. O., Boneti, J. A., Toni, C. A. G. \& Borin, J. B. (2018b). Integrating the pastoral component in agricultural systems. Revista Brasileira de Zootecnia. http://doi.org/10.1590/rbz4720170001.

Carvalho, P. C. F., Moraes, A., Pontes, L. S., Anghinonis, I., Sulc, M. \& Batello, C. (2014). Definições e terminologias para Sistema Integrado de Produção Agropecuária. Revista Ciência Agronômica, 45(5): 1040-1046. http://doi.org/10.1590/S1806-66902014000500020.

Chavez, L. F., Escobar, L. F., Anghinoni, I., Carvalho, P. C. \& Meurer, E. J. (2011). Diversidade metabólica e atividade microbiana no solo em sistema de integração lavoura-pecuária sob intensidades de pastejo. Pesquisa Agropecuária Brasileira. 46(10):1254-1261. http://doi.org/10.1590/S0100$204 X 2011001000020$.

Costa, A. N. L., Araujo, A. A. \& Araujo, E. P. (2016). Efeitos do estresse térmico na reprodução de fêmeas bovinas. Revista Brasileira Reprodução Animal, 40(4):123-125. Disponível em: http://www.cbra.org.br/portal/downloads/publicacoes/rbra/v40/n4/p123-125\%20(RB683).pdf.

Costa, D. F., Souto, D. V. O., Rocha, E. F., Guimaraes, L. J., Silva, M. R., Souza, B. B. \& Silva, G. A. (2015). Influência do estresse calórico na fisiologia hormonal de bovinos. Agropecuária científica no semiárido. 11(2):34-38. http://doi.org/10.30969/acsa.v11i2.662.

Costa, E. M. S., Dourado, L. R. B. \& Merval, R. R. (2012). Medidas para avaliar o conforto térmico em aves. Publicações em Medicina Veterinária e Zootecnia. 6(31). Disponível em: https://www.pubvet.com.br/uploads/fe2366b3bee041c79690dfe74f637380.pdf.

Costa, P. M. (2017). Desempenho produtivo de bovinos cruzados em sistemas de Integração Lavoura-Pecuária (Tese de Doutorado). Universidade Federal de Minas Gerais, Belo Horizonte, MG. Disponível em: https://repositorio.ufmg.br/handle/1843/BUOSARDEYP\#: :text=Reposit\%C3\%B3rio\%20Institucional\%20da\%20UFMG\%3A\%20Desempenho,sistemas\%20de\%20Integra\%C3\%A7\%C3\%A3o\%20Lavour a\%2DPecu\%C3\%A1ria\&text=Abstract $\% 3 \mathrm{~A}, \mathrm{de} \% 20$ corte $\% 20 \mathrm{em} \% 20$ sistemas $\% 20$ integrados.\&text=A\%20produtividade $\% 20$ na $\% 20$ recria $\% 20$ no,no $\% 20$ per $\%$ C3\%ADodo\% 20 de $\% 2011 \% 20$ meses.

Cunha, A. S., Rufino, L. M. A., Leite, R. C., Silva, M. X. \& Salvarani, F. M. (2018). Caracterização dos sistemas produtivos e dos produtores de leite da região Lago de Tucuruí, Pará, Brasil. Publicações em Medicina Veterinária e Zootecnia. 12(12):1-6. http://doi.org/10.31533/pubvet.v12n12a221.1-6.

Dias-Filho, M. B. \& Lopes, M. J. S. (2020). Histórico e desafios na pecuária bovina na Amazônia. Embrapa Amazônia Oriental. (documento 454). Belém, PA. Disponível em: https://www.embrapa.br/busca-de-noticias/-/noticia/55173092/publicacao-traca-a-historia-e-desafios-da-pecuaria-bovina-naamazonia\#: : :text=Foto\%3A\%20Reprodu\%C3\%A7\%C3\%A3o-

,Hist\%C3\%B3rico\%20e\%20desafios\%20da\%20pecu\%C3\%A1ria\%20bovina\%20na\%20Amaz\%C3\%B4nia\%2C\%20uma\%20publica\%C3\%A7\%C3\%A3o,e\% 20produtivo\%20dessa\%20cadeia\%20produtiva.

Euclides Filho, K. (2000). Produção de bovinos de corte e o trinômio genótipo-ambiente-mercado. Embrapa Gado de Corte. Campo Grande. Disponível em: https://www.embrapa.br/busca-de-publicacoes/-/publicacao/323697/producao-de-bovinos-de-corte-e-o-trinomio-genotipo---ambiente---mercado.

Euclides Filho, K., Figueiredo, G. R., Eucrides, V. P. B., Silva, L. O. C., Rocco, V., Barbosa, R. A. \& Junqueira, C. E. (2003). Desempenho de Diferentes Grupos Genéticos de Bovinos de Corte em Confinamento. Revista Brasileira de Zootecnia. 32(5): 1114-1122. http://doi.org/10.1590/S151635982003000500011 .

Façanha, D. A. E., Chaves, D. F., Morais, J. H. G., Vasconcelos, A. M., Costa, W. P. \& Guilhermino, M. M. (2013). Tendências metodológicas para avaliação da adaptabilidade a ambiente tropical. Revista Brasileira de Saúde e Produção Animal. 14(1): 91-103. Disponível em: https://www.scielo.br/j/rbspa/a/Kdf76XkztcjVvHRnVX8mVBx/abstract/?lang=pt\#: :text=Methodological\%20tendencies\%20of\%20adaptability\%20evaluatio n\%20to\%20tropical $\% 20$ environment\&text=Para\%20avalia\%C3\%A7\%C3\%A30\%20da\%20homeostase\%20recomenda,dentro\%20da $\% 20$ faixa\%20de $\% 20$ nor malidade.

Ferreira, D. P. (2020). Produção animal e características do pasto de capim piatã em sistema em integração após desbaste (Dissertação Mestrado). Faculdade de Agronomia e Zootecnia da Universidade Federal de Mato Grosso. Cuiabá, MT. Disponível em: https://www.ufmt.br/ppgat/images/uploads/Disserta\%C3\%A7\%C3\%B5es-Teses/Disserta\%C3\%A7\%C3\%B5es/2020/DISSERTA\%C3\%87\%C3\%83O\%20\%20DANIEL\%20PAULO.pdf.

Fialho, A. L. L., Souza-Cáceres, M. B., Silva, W. A. L., Arruda, E. D. S., Kischel, H., Ribeiro-Ferreira, M. G. C., Medeiros, C. F., Silva, J. R., Oliveira, M. V. M., Ferraz, A. L. J. \& Melo-Sterza, F. A. (2018). Efeito do estresse térmico calórico agudo e crônico sobre a qualidade oocitária de bovinos de raças adaptadas. Arquivo Brasileiro de Medicina Veterinária e Zootecnia. 70(1): 64-72. http://doi.org/ 10.1590/1678-4162-9494.

Fraser, M. D., Fleming, H. R., Moorby, J. M. (2014). Traditional vs Modern: Role of Breed Type in Determining Enteric Methane Emissions from Cattle Grazing as Part of Contrasting Grassland-Based Systems. PLOS ONE. 9. http://doi.org/10.1371/journal.pone.0107861.

Frizzo, A., Rocha, M. G., Restle, J., Montagner, D. B., Freitas, F. K. \& Santos, D. T. (2003). Suplementação Energética na Recria de Bezerras de Corte Mantidas em Pastagem de Inverno. Revista Brasileira de Zootecnia. 32(3): 643-652. http://doi.org/10.1590/S1516-35982003000300016.

Figueiredo, D. M., Oliveira, A. S., Sales, M. F. L., Paulino, M. F. \& Vale, S. M. L. R. (2007) Análise econômica de quatro estratégias de suplementação para recria e engorda de bovinos em sistema pasto-suplemento. Revista Brasileira de Zootecnia. 36(5):1443-1453. Disponível em: https://www.rbz.org.br/ptbr/article/analise-economica-de-quatro-estrategias-de-suplementacao-para-recria-e-engorda-de-bovinos-em-sistema-pasto-suplemento/. 
Galloso-Hernández, M. A., Rodríguez-Estévez, C., Soca-Pérez, M., Dublin, D., Iglesias-Gómez, J. \& Guelmes, L. S. (2020). Effect of Silvopastoral Systems in the Thermoregulatory and Feeding Behaviors of Water Buffaloes Under Different Conditions of Heat Stress. Frontiers in Veterinary Science. 7. http://doi.org/10.3389/fvets.2020.00393.

Gasparini, L. V. L., Costa, T. S., Hungaro, O. A. L., Sznitowski, A. M. \& Vieira Filho, J. E. R. (2017). Sistemas integrados de produção agropecuária e inovação em gestão: estudos de casos no Mato Grosso. Rio de Janeiro: Instituto de Pesquisa Econômica Aplicada - IPEA. Disponível em: https://www.ipea.gov.br/portal/index.php?option=com_content\&view=article\&id=29871.

IBGE. (2018). Instituto Brasileiro de Geografia e Estatística. Brasília: IBGE.

Lima. M. C. D. \& Gama, D. C. (2018). O sistema de integração lavoura-pecuária-floresta no brasil: conceitos, desafios e novas perspectivas. Agroforestalis News. 3(1). Disponível em: https://seer.ufs.br/index.php/AGRO/article/view/9752.

Macedo, G. G., Costa e Silva, E. V., Martins, L. F., Pinho, R. O. \& Monteiro, B. M. (2013). Estresse por calor: alterações na fisiologia reprodutiva e estratégias para amenizar seus efeitos negativos na fertilidade de fêmeas bovinas. Arquivos de Ciências Veterinárias e Zoologia da UNIPAR. 16(1): 79-85. Disponível em: https://revistas.unipar.br/index.php/veterinaria/article/view/4487.

Macedo, M. C. M. \& Araujo, A. R. (2012). Sistemas de integração lavoura-pecuária: alternativas para recuperação de pastagens degradadas. In: Bungenstab, D. J. Sistemas de integração lavoura-pecuária-floresta: a produção sustentável. (2a. ed., pp. 27-48). Brasília: Embrapa. Disponível em: https://www.embrapa.br/busca-de-publicacoes/-/publicacao/938814/sistemas-de-integracao-lavoura-pecuaria-floresta-a-producao-sustentavel.

Marconi, M. A. \& Lakatos, E. M. (2012). Metodologia do trabalho científico: procedimentos básicos, pesquisa bibliográfica, projeto e relatório, publicações e trabalhos científicos. (7. ed.). São Paulo: Atlas.

Melo, A. F., Moreira, J. M., Ataides, D. S., Guimaraes, R. A. M., Loiola, J. L. \& Sardinha, H. C. (2016). Efeitos do estresse térmico na produção de vacas leiteiras: Revisão. Publicações em Medicina Veterinária e Zootecnia. 10(10): 721-730. http://doi.org/10.22256/pubvet.v10n10.721-730.

Moraes, A., Carvalho, P. C. F., Pelissari, A., Anghinonni, I., Lustosa, S. B. C., Lang, C. R., Assmann, T. S., Deiss, L. \& Nunes, P. A. A. (2018). Sistemas integrados de produção agropecuária: conceitos básicos e históricos no Brasil. In: Souza, E. D., Silva, F. D., Assmann, T. S., Carneiro, M. A. C., Carvalho, P. C. F. \& Paulino, H. B. Sistemas integrados de produção agropecuária no Brasil. (1a. ed., pp. 13-28).Tubarão- Copiart.

Nicodemo, M. L. F., Garcia, A. R., Silva, V. P. \& Paciullo, D. S. C. (2018). Desempenho, saúde e conforto animal em sistemas silvipastoris no Brasil. São Carlos, SP: Embrapa Pecuária Sudeste. Disponível em: https://www.embrapa.br/busca-de-publicacoes/-/publicacao/1095705/desempenho-saude-e-confortoanimal-em-sistemas-silvipastoris-no-

brasil\#: :text=Desempenho\%2C\%20sa\%C3\%BAde\%20e\%20conforto\%20animal\%20em\%20sistemas\%20silvipastoris\%20no\%20Brasil.,-

Autoria\%3A\%20NICODEMO\%2C\%20M.\%20L.\%20F.\&text=Bovinos\%2C\%20por\%20exemplo\%2C\%20estar\%C3\%A3o\%20confort\%C3\%A1veis,1.000\%2 $0 \mathrm{~W} \% 2 \mathrm{Fm} \% \mathrm{C} 2 \% \mathrm{~B} 2 \% 2$ Fhora.

Nogueira, E., Oliveira, L. F., Nicacio, A. C., Gomes, R, C. \& Medeiros, S. R. (2015). Nutrição aplicada à reprodução de bovinos de corte. Embrapa Gado de Corte. Disponível em: https://www.embrapa.br/busca-de-publicacoes/-/publicacao/1011240/nutricao-aplicada-a-reproducao-de-bovinos-de-corte.

Ohashi, O. M., Cordeiro, M. S., Santos, S. S. D., Almeida, N. N. C., Silva, T. V. G. \& Rolim Filho, S. T. (2018). Desafio da Pecuária na Amazônia frente ao novo código florestal brasileiro. Revista Brasileira Reprodução Animal. 42(3-4): 202-205. Disponível em: http://www.cbra.org.br/portal/downloads/publicacoes/rbra/v42/n3-4/p202-205\%20(RB762).pdf.

Oliveira, E. C., Delgado, R. C., Rosa, S. R., Sousa, P. J. O. P. \& Neves, L. O. (2013). Efeitos do estresse térmico sobre a produção de bovinos de Leite no município de Marilândia-ES. Enciclopédia Biosfera: Centro Científico Conhecer. 9(16):913-921. Disponível em: https://www.conhecer.org.br/enciclop/2013a/agrarias/EFEITO\%20DO\%20ESTRESSE\%20TERMICO.pdf.

Paciullo, D. S., Carvalho, C. A. B., Aroeira, L. J. M., Morenz, M. J. F., Lopes, F. C. F. \& Rossiello, R. O. P. (2007). Morfofisiologia e valor nutritivo do capim braquiária sob sombreamento natural e a sol pleno. Pesquisa Agropecuária Brasileira. 42(4): 573-579. https://doi.org/10.1590/S0100-204X2007000400016.

Paciullo, D. S., Lopes, F. C. F., Malaquias, J. D., Viana Filho, A., Rodriguez, N. M., Morenz, M. J. \& Aroeira, L. J. M. (2009). Características do pasto e desempenho de novilhas em sistema silvipastoril e pastagem de braquiária em monocultivo. Pesquisa Agropecuária Brasileira, Brasília. 44(11): 1528-1535. https://doi.org/10.1590/S0100-204X2009001100022.

Paim, T. P., Goulart, R. O., Silva, D. A. L., Sousa, J. A., Gonçalves, L. F., Claudio, F. L., Calgaro Junior, G., Gleria, A. A., Santos, L. C. \& Alves, E. M. (2018). Relationship of weight gain with infrared temperatures in Nelore and F1 (Nelore $\times$ Angus) heifers reared in two forage production systems. Journal of Animal Science, Oxford University Press. 96(9): 4002-4011. https://doi.org/10.1093/jas/sky242.

Pariz, C. M., Andreotti, M., Tarsitano, M. A. A., Bergamaschine, A. F., Buzetti, S. \& Chioderoli, C. A. (2009). Desempenhos técnicos e econômicos da consorciação de milho com forrageiras dos gêneros Panicum e Brachiaria em Sistema de integração lavoura-pecuária. Pesquisa Agropecuária Tropical. 39(4): 360-370. https://doi.org/10.5216/pat.v39i4.5651.

Rocha. D. R., Salles, M. G. F., Moura, A. A. A. N. \& Araújo, A. A. (2012). Impacto do estresse térmico na reprodução da fêmea bovina. Revista Brasileira Reprodução Animal. 36(1):18-24. Disponível em: http://www.cbra.org.br/pages/publicacoes/rbra/v36n1/pag18-24.pdf

Santos, D. B., Vanin, J., Silva, C. G., Bondan, C. \& Bortoluzzi, C. (2013). Qualidade do leite de propriedades familiares praticantes de integração lavourapecuária em função do uso do solo. Arquivo Brasileiro de Medicina Veterinária e Zootecnia. 65(4): 1217-1222. https://doi.org/10.1590/S010209352013000400038 .

Santos, P. E. F., Eustaquio Filho, A. \& Yamamoto, S. M. (2008). Desempenho de fêmeas bovinas em pastejo e confinamento. Publicações em Medicina Veterinária e Zootecnia. Disponível em: http://www.pubvet.com.br/material/Santos295.pdf.

Santos, B. R. (2011). Modelo de gestão estratégica para propriedades dedicadas à bovinocultura de corte (Monografia de graduação). Universidade Federal de Roraima, RR. 
Research, Society and Development, v. 10, n. 16, e498101624014, 2021

(CC BY 4.0) | ISSN 2525-3409 | DOI: http://dx.doi.org/10.33448/rsd-v10i16.24014

Schutz K. E., Rogers, A. R., Cox, N. R., Webster, J. R. \&Tucker, C. B. (2011). Dairy cattle prefer shade over sprinklers: Effects on behavior and physiology. Journal of Dairy Science. 94(1): 273-283. https://doi.org/10.3168/jds.2010-3608.

Silva, J. S., Santos, D. T. \& Vieira, P.C. (2016). Integração lavoura pecuária floresta (ILPF). Boletim Técnico Informativo. Rio Grande do Sul: Plano ABC. Disponível em: https://www.agricultura.rs.gov.br/upload/arquivos/201608/01145315-boletim-tecnico-informativo-integracao-lavoura-pecuaria-floresta.pdf

Silva, L. F. M. (2017). Desempenho e características da carcaça de novilhas de três grupos genéticos recriadas em pastagem e terminadas em confinamento (Dissertação de Mestrado). Universidade de Brasília, Brasília. Disponível em: https://repositorio.unb.br/handle/10482/23759.

Silva, W. A. L., Poehland, R., Oliveira, C. C., Ferreira, M. G. C. R., Almeida, R. G., Caceres, M. B. S., Macedo, G. G., Silva, E. V. C., Alves, F. V., Nogueira, E. \& Melo-Sterza, F. A. (2020). Shading effect on physiological parameters and in vitro embryo production of tropical adapted Nellore heifers in integrated crop-livestock-forest systems. Tropical Animal Health and Production. 52: 2273-2281. https://doi.org/10.1007/s11250-020-02244-3.

Soares, A. B., Missio, R. L., Schmitt, D., Aiolfi, R. B. \& Deifeld, F. L. C. (2018). Componente animal em sistemas integrados de produção agropecuária. In: Souza, E. D., Silva, F. D., Assmann, T. S., Carneiro, M. A. C., Carvalho, P. C. F. \& Paulino, H. B. (ed). Sistemas integrados de produção agropecuária no Brasil (1a. ed., pp.187-210). Tubarão: Copiart.

Vilela, L., Martha Junior, G. B., Macedo, M. C. M., Marchao, R. L., Guimaraes Junior, R., Pulrolnik, K. \& Maciel, G. A. (2011). Sistemas de integração lavoura-pecuária na região do Cerrado. Pesquisa Agropecuária Brasileira, 46(10): 1127-1138. Disponível em: https://www.scielo.br/j/pab/a/Bc4Wp3CY9494yN9zdHzNGBP/?lang=pt.

Ximenes, L. J. F. \& Martins, G. A. (2018). Bovinocultura leiteira: melhoramento genético econômico (52). Escritório Técnico de Estudos Econômicos do Nordeste - ETENE. Disponível em: https://www.bnb.gov.br/s482-dspace/handle/123456789/366 